\title{
PARTICIPACIÓN COMUNITARIA Y LEGISLACIÓN EN EL SALVADOR. ANÁLISIS DE LA LEGISLACIÓN IDENTIFICADA
}

\author{
Guillermo Cortez Juárez ${ }^{1}$ \\ Funcionario del Ministerio de Educación de El Salvador \\ El Salvador
}

Recibido 01 de setiembre 2007 - Aprobado 31 de octubre 2007

\begin{abstract}
Resumen: La participación comunitaria es estratégica para lograr efectividad en los procesos de cambio político, económico, social, educativo y cultural; en tanto garantiza la efectividad, calidad y sostenibilidad de las políticas sociales. Por ello, desde la legislación del estado nacional, debe estimularse y promocionarse dicha participación, se dice que siempre ha estado presente, tradicional e históricamente, ha sido impositiva y antidemocrática.

La Educación como acto esencialmente humano, es un instrumento de primer orden que las sociedades deben aprovechar para desarrollar y estimular la participación democrática. Por eso, la educación puede constituirse en una nueva cultura: la de la participación democrática como camino indispensable para el fortalecimiento y desarrollo de la cultura de Paz.
\end{abstract}

Palabras clave: Promoción, participación comunitaria, legislación, cultura de paz.

Abstract: Community participation has been considered strategic in order to achieve effectiveness in political, economical, social, educational and cultural change processes as a mean of guarantying effectiveness, quality and sustainability in social policy. Accordingly, this participation must be stimulated and promoted through national legislation since it has traditionally and historically been imposed and undemocratic.

Education, as an essentially human act, is a first order instrument that societies must take advantage of in order to develop and stimulate democratic participation. Therefore, education may represent a new culture: one of democratic participation as a fundamental way to empower and develop a Peace culture.

Key words: Promotion, community participation, legislation, peace culture.

\section{INTRODUCCIÓN}

La participación comunitaria se ha considerado estratégica la efectividad de los procesos de cambio en diferentes áreas del quehacer humano: en lo político, económico, social, cultural,

Salvadoreño, Profesor de Educación Básica, Licenciado en Psicología y M.Sc. en Derechos Humanos y Educación para la Paz. Actualmente trabaja en el Ministerio de Educación de El Salvador. Esudiante de la Maesría en Educación Rural Centroamericana. Correo electrónico: guicoju@yahoo.com 
educativo, etcétera. Esto parece muy importante, y sin embargo, en El Salvador, no existe una legislación que promueva y estimule la participación comunitaria.

En la Constitución de la República, el artículo 7 "Derechos individuales", se afirma expresamente:

Los habitantes de El Salvador tienen derecho a asociarse libremente y a reunirse pacíficamente y sin armas para cualquier objeto lícito. Nadie podrá ser obligado a pertenecer a una asociación. No podrá limitarse ni impedirse a una persona el ejercicio de cualquier actividad lícita, por el hecho de no pertenecer a una asociación, se prohíbe la existencia de grupos armados de carácter político, religioso o gremial (República de El Salvador. Asamblea Legislativa de El Salvador, 1983).

Con este artículo, pareciera que constitucionalmente se le otorga un fundamento legal a la participación, pero debe entenderse que se refiere a la participación en acciones lícitas, las cuales aunque correspondan a programas o proyectos de beneficio comunitario; y son vistas como ilícitas cuando son promovidas por sectores opositores al gobierno.

De manera más clara y específica, la Constitución de la República de El Salvador no promueve ni estimula la participación comunitaria como estrategia para diseñar, implementar, ejecutar y evaluar proyectos de desarrollo social. Seguramente hay razones para no hacerlo, pero son ignoradas públicamente. En todo caso, ello no niega la importancia de estimularla y promocionarla como estrategia para garantizar la efectividad, y la calidad de las políticas sociales.

Revisando el marco jurídico del país, no se encuentran en ninguna ley, reglamento o normativa vigente, aspectos relacionados con el tema de la promoción de la participación; lo cual no significa que se obvie su importancia capital para lograr la efectividad de programas o proyectos. Sin embargo, en general, se puede afirmar que no existe claridad metodológica entre los diseñadores y ejecutores de programas o proyectos, sobre cómo desarrollar y fortalecer una cultura de participación democrática en las comunidades. Como consecuencia, muy raras veces se habla de programas o proyectos efectivos, pues generalmente, no resuelven los problemas o necesidades que se supone les dieron origen.

En el campo de la educación es común observar este fenómeno; por ejemplo, se han impulsado programas y proyectos para erradicar el analfabetismo, pero por ahora, los habitantes de pueblos, caseríos y ciudades deben continuar soportando las consecuencias que su presencia genera en su vida. De igual manera, se han desarrollado muchos programas para mejorar la calidad de la educación, en los cuales, se han invertido fuertes cantidades de dinero; pero la sociedad latinoamericana sigue padeciendo las consecuencias del subdesarrollo producto, en parte, de la poca o baja calidad educativa que reciben niñas, niños y jóvenes.

A pesar de todo, la comunidad salvadoreña participa en los procesos de cambio. De hecho, la participación comunitaria siempre ha estado presente, de una manera u otra; el problema es que, generalmente, a la comunidad se le utiliza o se le impone la participación para que pueda gozar de determinados beneficios. Por ejemplo, en un proyecto de introducción de agua potable a una comunidad, el ciudadano que no participa no tiene derecho a disfrutar de los beneficios del proyecto. Por lo tanto, no es que se le brinden los espacios de participación, sino que participa cuando los responsables consideran conveniente.

En relación con los proyectos de desarrollo rural, los mecanismos de participación son iguales. Tanto los organismos no gubernamentales como los gubernamentales, empresas 
privadas, municipalidades, clubes de servicio y otros; funcionan con el mismo esquema de participación: a) diseñan el proyecto desde fuera de la comunidad, b) informan a unos cuantos miembros de la comunidad lo que se va a hacer, c) ejecutan el proyecto, d) muy raras veces lo evalúan, e) no promueven ni estimulan la participación, porque esto no es visto como una estrategia de calidad, efectividad y sostenibilidad; tampoco como un derecho que tiene la comunidad para su auto desarrollo, sino como una dádiva que corresponde a la generosidad de quienes diseñan y ejecutan estas propuestas, o como un deber que las personas han de cumplir para acceder a ciertos derechos.

\section{LA LEGISLACIÓN SALVADOREÑA Y EL TEMA DE LA PARTICIPACIÓN}

De la legislación salvadoreña se ha revisado la Constitución de la República (que es nuestra Carta Magna), el Código de Trabajo, el Código de Familia, la Ley General de Educación, la Ley de la Carrera Docente y la Convención de los Derechos del Niño; y se puede afirmar que se refieren a la participación en términos muy generales, pero se conoce de mecanismos para su difusión y montos presupuestarios para ello.

En el artículo que se refiere a los fines de la Educación, establecidos en la Constitución de El Salvador, Sección tercera: Educación, Ciencia y Cultura, se dice, Art. 55:

La Educación tiene los siguientes fines: lograr el desarrollo integral de la personalidad, en su dimensión espiritual, moral y social; contribuir a la construcción de una sociedad democrática, más próspera, justa y humana, inculcar el respeto a los derechos humanos y la observancia de los correspondientes deberes, combatir todo espíritu de intolerancia y de odio, conocer la realidad nacional e identificarse con los valores de la nacionalidad salvadoreña; y propiciar la unidad del pueblo centramericano (República de El Salvador. Asamblea Legislativa de El Salvador, 1983).

Podrá comprenderse que para lograr tales fines educativos, se requiere de una estrategia que se fundamente en la participación de los ciudadanos.

Es muy difícil construir democracia sin una participación consciente de la ciudadanía y, de igual manera, es casi imposible construir una sociedad justa, más humana y próspera sin considerar que los constructores de dicha sociedad son los mismos ciudadanos, a quienes en consecuencia, se les deben brindar espacios y estimular la participación activa y solidaria.

En la Ley General de Educación se asume el mandato constitucional en relación con las finalidades de la educación; pero en ella, tampoco se reconoce la importancia de promover y desarrollar un buen sistema de participación democrática que incluya a todos los sectores sociales vinculados con la educación del país. La ley se refiere más bien a la participación de los estudiantes, los maestros y los padres de familia. Estos, según el artículo 93:

Deberán involucrarse responsablemente en la formación de sus hijos y en el reforzamiento de la labor de la escuela, con el propósito de propiciar el desarrollo de los buenos hábitos, la disciplina, la autoestima, los valores, el sentido de pertenencia, y solidaridad y la personalidad en general (Ministerio de Educación, 1994-1999, p. 14). 
En el artículo 69 de dicha ley, en lo referente a la Supervisión Educativa, se establece que: "La Supervisión Educativa tiene los objetivos siguientes: (...): “b) Formular y orientar la participación activa de la comunidad para el suministro de los servicios educativos (...)" (Ministerio de Educación, 1994-1999, p. 11). Aunque no se concretiza el concepto de comunidad, se entiende que se refiere en términos amplios a los padres de familia, docentes y estudiantes.

Para normar la participación de dichos actores, existen normativas específicas referidas especialmente a tres diferentes modalidades de administración en las que descansa la gestión escolar. En ellas se orienta sobre cómo deben organizarse, qué requisitos deben cumplir para legalizarse y cómo deben administrar los recursos del centro.

Las Asociaciones Comunales para la Educación, más conocida como Educación con Participación de la Comunidad (EDUCO); es una modalidad caracterizada por conformarse exclusivamente por madres y padres de familia, y son ellos, en coordinación con el director del centro escolar, los responsables del funcionamiento del centro.

Los Consejos Directivos Escolares (CDE) son una modalidad de administración colegiada. Está conformada por El Director o Directora, representantes de los docentes, de los padres y madres de familia, y por representantes de los estudiantes.

Los Centros Educativos Católicos Escolares (CECE) también poseen su modalidad de administración, auque está conformada por diferentes actores educativos como el director, los docentes, padres de familia, estudiantes y ex alumnos, y mantiene un sistema de participación centralizada por el director del centro, que normalmente es una religiosa, un religioso o el párroco de la comunidad.

La gestión educativa de estas modalidades se fundamenta en la participación comunitaria; sin embargo, ésta es mediatizada por las unidades técnico-administrativas del Ministerio de Educación; desde allí se norma quiénes participarán, cómo lo harán y en qué. Es interesante observar que a las comunidades se les permite participar, sobre la base del cumplimiento de normativas específicas en aspectos de orden administrativo y logístico, pero no en aspectos que tienen que ver con la misión fundamental de la escuela, como en la definición de los contenidos de la educación que reciben sus hijos.

Sin descartar la importancia que la participación tiene, el método para lograrla es más impositivo que democrático; por ello, no es difícil encontrar personas que manifiestan sentirse manipuladas en su participación u obligadas a hacerlo, y en consecuencia, la participación pierde su verdadero sentido, pues los padres no logran apropiarse de su rol como actores educativos efectivos.

A pesar que no existe un involucramiento pleno de las comunidades en el proceso educativo, debido en gran medida a la concepción de la participación impositiva, se ha podido descubrir, según Mejía (2007) que las estrategias educativas actuales tienden a ser más efectivas en la medida que se les asigna un carácter más participativo, ya que los resultados son mejores cuando los beneficiarios se involucran en las decisiones desde la planificación de la estrategia, acompañan en el proceso y son actores en su implementación.

¿Por qué debe ser así? Muchos estudios en el ámbito latinoamericano, han demostrado que a mayor participación de los padres en el proceso educativo de sus hijos, mayor es el rendimiento académico de estos; sin embargo, es necesario insistir en que la participación, si bien está orientada más a lo administrativo, debe abrirse el campo en lo pedagógico y curricular.

Según Mejía (2007) en el caso de una comunidad rural por ejemplo los habitantes tienen conocimiento pleno de su medio y de la forma compleja en que evoluciona su vida para lograr el sustento diario de su familia, conservar su identidad, su cultura y poder convivir con los demás; realizan trueques e intercambios en sus bienes, forman redes de información, todos saben de todos. El 
conocimiento de estos factores son claves para optimizar los recursos con que cuenta una institución escolar que generalmente son pocos, y mucho menos cuando se trata de una escuela rural por ello su participación es importantísima para optimizar el uso de los pocos recursos con que cuentan.

Es importante recalcar que para hablar de calidad de la educación, hay que hablar de equidad, lo que lleva a reflexionar acerca de que la calidad está estrechamente relacionada con el grado de participación de la comunidad en la gestión educativa que, según Durston, se da en dos planos: "la comunicación intercultural y los contratos entre autores en el sistema escuela-comunidad (Durston, 1997, p. 68)". Según Mejía "Cuando se atiende estas dos dimensiones, se logra la eficacia en lo pedagógico, la ética y transparencia en todas las acciones que realizan los involucrados, tanto de la escuela como de la comunidad, se desarrolla positivamente la autoestima personal. Es toda una sinergia en el sistema educativo de la escuela, la cual, se ve reflejada en los productos o aprendizajes significativos que adquieren los estudiantes" (Mejía, 2007, p. 1).

Cuando esto no sucede, generalmente se evaden responsabilidades de la baja calidad de la educación en los sectores rurales con posiciones deterministas, al relacionar directamente el logro educativo con las condiciones socioeconómicas del hogar del estudiante. Gracias al desarrollo de la investigación científica en el ámbito educativo, este determinismo empieza a superarse con nuevas relaciones entre el medio sociocultural del estudiante y el logro escolar, como la participación familiar y comunitaria, una pedagogía moderna, mayor autonomía de la educación descentralizada, mayor responsabilidad de los docentes, reconocimiento del potencial comunitario, e interacción de culturas entre la escuela y el medio local para que surja una nueva cultura.

La Ley General de Educación también hace referencia a la participación de las niñas y los niños en el proceso educativo, pero no lo hace desarrollando previamente procesos de sensibilización sobre la necesidad de participar en dicho proceso. Más bien, lo hace desde el enfoque de los deberes y derechos. En el Capítulo II de esta ley, referido a los educandos, se establece: Art. 89: "Son deberes de los educandos (...): a) participar en actividades de enseñanza y de formación que desarrolle la institución en la que está inscrito (...) (Ministerio de Educación, 1994-1999, p. 13)” y por otra parte en el Art. 90 , se establece que son derechos de los educandos (...): "f) participar en conformación y actividades del consejo de alumnos y ser electo democráticamente como miembro del Consejo Directivo Escolar del centro educativo donde estudia (...)" (Ministerio de Educación, 1994-1999, p. 14).

La participación legal de los estudiantes se convierte en una participación antidemocrática e impositiva que se reduce a aspectos organizativos para desarrollar acciones diseñadas por los docentes, y en las que ellos no tienen voz ni voto, tales como: proyectos de limpieza, proyectos de convivencia (reglamento escolar), proyectos de huertos caseros, proyectos de ornato y limpieza en el centro, etcétera.

Aunque la ley favorece la participación del estudiante en organizaciones estudiantiles o en organismos administrativos, resulta difícil desarrollar procesos democráticos de elección de los miembros del consejo estudiantil, porque los estudiantes no han desarrollado experiencias democráticas en la vida cotidiana de la escuela. Esto hace reflexionar sobre la necesidad de democratizar, por un lado, la vida de la escuela y la enseñanza, y por otro, la necesidad de formar maestros no solo con mentalidad democrática, sino con vivencias y cultura democrática.

Es obvio que las niñas y los niños participan en actividades de la escuela en las cuales se les asignan algunas responsabilidades; sin embargo, no se les propone, sino al contrario, se les impone. Aunque las actividades impuestas hayan sido diseñadas atendiendo lo estipulado en convenciones, tratados, leyes o reglamentos de atención y protección al menor u otros, no las exime del carácter 
impostivo. Los estudiantes por ejemplo, no participan en la definición de las normas de convivencia que se deben observar para lograr un ambiente armónico en la escuela; pero sí se les invita a cumplir el reglamento elaborado sin su consentimiento y se les informa de los castigos que deberán recibir si no acatan las normas establecidas.

La Ley General de Educación también se refiere a la participación de los docentes en el proceso educativo. Para ello, se ha creado la Ley de la Carrera Docente y su respectivo reglamento. Según el art. 1 de dicha ley, el objeto es: "Regular las relaciones del Estado y de la comunidad educativa con los educadores al servicio del primero, de las instituciones autónomas, de las municipales y de las privadas; así como valorar sistemáticamente el escalafón, tanto en su formación académica, como en su antigüedad" (Ministerio de Educación, 1994-1999, p. 1).

En ella, se establecen las funciones del docente, sus derechos y deberes, así como el régimen disciplinario y el funcionamiento de tribunales especiales para dirimir los problemas entre maestros o entre estos, los alumnos y alumnas con los padres y madres de familia.

En toda la legislación salvadoreña se observa la ausencia de instancias para promover la participación democrática de los diferentes actores; tampoco se han estipulado presupuestos específicos para ello; pero sí se encuentran responsabilidades asignadas y se ha previsto un régimen de sanciones para el incumplimiento de dichas funciones. Sobre este aspecto, es importante decir que la inexistencia de una cultura de exigibilidad ha permitido la ausencia de instituciones responsabilizadas de la promoción y la estimulación de la participación democrática de las comunidades.

Un aspecto importante de reflexionar, es la viabilidad de la participación democrática en El Salvador. Al respecto, se puede afirmar que sí es viable; entendiéndose la participación como el involucramiento libre, consciente y decidido en los programas o proyectos de desarrollo comunitario, lo cual pasa por el empoderamiento que la comunidad debe hacer de ellos, mediante la participación en actividades de sensibilización sobre la necesidad de aportar, solidariamente, al proceso de desarrollo comunitario.

De hecho “...todos los estudios y análisis sobre la realidad nacional concluyen que, sin una educación instalada en el centro de nuestra problemática histórica, es imposible avanzar efectivamente hacia el desarrollo. La educación es el instrumento social fundamental para cambiar, de manera definitiva, las condiciones de vida de la población, en sus distintos segmentos, así como para desarrollar nuestra cultura propia, en armonía con la cultura universal. Sólo la educación es capaz de superar en profundidad estructural, la marginación sociocultural que ha dificultado sistemáticamente, hasta la fecha, una evolución articulada, armoniosa y consistente en el país" (Comisión Presidencial para el Desarrollo de la Sociedad del Conocimiento, 2004, p. 5).

Por eso, la misma Comisión reafirma que:

Los procesos educativos tienen que fortalecer la cultura democrática y de paz, desarrollando formas y niveles de convivencia que nos impulsen a convivir armónicamente con base a normas de ordenamiento jurídico e institucional, conocidas y aceptadas por todos. La formación de una conciencia ética, el cultivo de valores morales, el desarrollo de la capacidad crítica, la transparencia y la responsabilidad como elementos básicos de la actividad social son elemento clave de la cultura democrática que deben estar presentes en la escuela, tanto en los procesos educativos como en el funcionamiento institucional (Comisión Presidencial para el Desarrollo de la Sociedad del Conocimiento, 2004, p. 11). 
En consonancia con Arrien (2003), nuestro país debe apostarle a una educación que parta de la vida de las personas, de su cultura, de sus aspiraciones, de sus necesidades y de sus limitaciones, para hacerlas más humanas. Por ello, desde el inicio de su proceso formativo, los niños y las niñas deben educarse en formas de participación que incluyan elecciones y representación, capacidad de negociación y rechazo de formas de proceder autoritarias y violentas.

La asimilación de una cultura de paz, entendimiento y respeto, fortalece y arraiga la cultura democrática. El amor a la vida, el rechazo de la violencia, el cultivo de la tolerancia y la capacidad de diálogo, el desarrollo de los derechos humanos, de la solidaridad, el cuidado de la naturaleza, y la liberación de la propia generosidad frente a tareas derivadas de estos principios forman parte de la cultura de paz.

Particularmente para El Salvador, considerado como uno de los países más violentos del mundo, estos aspectos deben ser considerados a la hora de replantear la educación. Los altos índices de violencia, las altas tasas de desempleo y subempleo, la marginación y la exclusión social que alimenta la emigración hacia el extranjero, la cultura de la anarquía, la carencia de institucionalidad democrática y jurídica, constituyen serios desafíos, urgentes de resolver mediante un nuevo modelo educativo que debe ser diseñado y ejecutado con la participación de todos los sectores productivos del país, las iglesias y demás instituciones sociales, políticas y culturales.

La educación es, en este sentido, el instrumento de primer orden para generar una nueva cultura: la cultura de la participación democrática y de la paz. Através de ella se puede generar el cambio para pasar de una sociedad autocrática a una democrática; es decir, se trata de generar y crear una nueva cultura: la de la participación democrática.

Por ahora, la educación tiene una deuda, pues a juicio de (Arrien, 2003, pp. 3-4) “...no ha cumplido con su papel original de evitar la desacelerada deshumanización del ser humano, llevándolo a convertirse en un bien económico". Por ello, es necesario un replanteamiento radical de la educación de acuerdo con las ideas que se han expuesto.

En materia curricular se observa un avance muy significativo en la propuesta que se establece desde la oficialidad, pues constitucionalmente, se establece, en el artículo 57, Sección tercera: Educación, Ciencia y Cultura que: “...la enseñanza que se imparta en los centros educativos oficiales será esencialmente democrática" (República de El Salvador. Asamblea Legislativa de El Salvador, 1983). Ello supone formar ciudadanos con mentalidad democrática y con cultura participativa, para lo cual, se deben realizar transformaciones en el sistema educativo, fundamentadas en los principios de la autonomía, la pertinencia, la equidad de género la calidad, la equidad, y la solidaridad.

Se requiere además rediseñar un currículo de manera que responda a las necesidades de las y los estudiantes en las circunstancias históricas actuales. Un currículo que favorezca el rescate y desarrollo de los diferentes grupos étnicos y autóctonos, considerando la sugerencia de tratar de formar al ciudadano para que contribuya al desarrollo de la comunidad, y de igual manera, retomando la experiencia de rediseñar un sistema educativo que considere las características naturales y sociales de la región (multiétnico, multilingüe y multicultural).

A los obreros de la educación, a los que estimulan la generación de aprendizajes significativos en niños y jóvenes, se les debe incorporar en un nuevo proceso de formación que incluya la formación inicial y la formación en servicio, o sea, un nuevo sentido de la profesionalización docente.

Lo que se buscaría es contar con un nuevo tipo de maestro al estilo de como lo planteaba el Dr. Rafael Lucio Gil, en su obra: Políticas Públicas para el Desarrollo Rural (Gil, 2007), un maestro con una competencia real que incluya: capacidad comunicativa, capacidad para empatizar con la comunidad educativa a la que servirá y capacidad para desarrollar un liderazgo propositivo. 
Para ello, se deben buscar las estrategias necesarias para que en su proceso de profesionalización se desarrollen la inteligencia emocional y la inteligencia racional.

La construcción de una nueva sociedad formada por nuevos ciudadanos, tal como lo plantea la Comisión Presidencial para el desarrollo de la Sociedad del Conocimiento (2004) en su propuesta "Educar para el País que Queremos", implica construir una educación de primer nivel para todos los salvadoreños y salvadoreñas, independientemente de su ubicación geográfica o su condición socioeconómica.

En este marco, se plantean cuatro objetivos fundamentales:

a) Asegurar la formación integral de las personas.

b) Lograr 11 grados de escolaridad para toda la población.

c) Crear oportunidades flexibles de formación profesional y técnica después del noveno grado.

d) Fortalecer la investigación, la ciencia y la tecnología en función de la productividad, el bienestar social y el desarrollo cultural.

Siendo la educación un proceso socialmente compartido, debe promover y estimular la participación de la comunidad como estrategia válida para darle cumplimiento a los fines de la educación que la constitución de la República salvadoreña establece.

\section{REFERENCIAS}

Arrien, J. B. (2003). La educación Rural en Centroamérica (Conferencia Inaugural). En M. E. Aguilar y Cerdas (Comp.), La educación rural en Centroamérica. II Seminario centroamericano de educación rural (pp. 1-23). Heredia: EUNA.

Comisión Presidencial para el desarrollo de la Sociedad del Conocimiento (2004). Educar para el país que queremos. San Salvador, El Salvador.

Durston, J. (1997). La participación comunitaria en la gestión de la escuela rural. En E. Cohen (Aut.), Educación, eficiencia y equidad (pp. 68-84). Santiago, Chile: Ediciones SUR, 1997.

Gil, R. (2007). Políticas públicas para el desarrollo rural, el caso de Nicaragua. [Ponencia etapa presencial de la maestría en educación rural”. León, Nicaragua.

Mejía, N. (Febrero de 2007). Promoción de la participación en comunidad con especificidad en el trabajo con instituciones, Ensayo. Heredia: Maestría Centroamericana en Educación Rural.

Mendoza, R. (1995). Código de familia, convención sobre los derechos del niño, y documentos complementarios. San Salvador: Editorial Jurídica Salvadoreña.

Ministerio de Educación. (1994-1999). Ley de la carrera docente. Decreto 665. San Salvador.

Ministerio de Educación. (1994-1999). Ley General de Educación. Decreto 917. San Salvador. 
Ministerio de Educación. (2004). Normativas y Procedimientos para el Funcionamiento del Consejo Directivo Escolar. San Salvador: Ministerio de Educación.

Ministerio de Educación. (2004). Normativas y Procedimientos para el Funcionamiento de la Asociación Comunal para la Educación. San Salvador: Ministerio de Educación.

Ministerio de Educación. (2004). Normativas y procedimientos para el funcionamiento de los centros educativos católicos escolares. San Salvador: Ministerio de Educación.

República de El Salvador. Asamblea Legislativa de El Salvador (1983). Constitución de la República de El Salvador. San Salvador, El Salvador, C.A. 